\title{
Transcorporeal Selective Decompression without Fusion for Midline-located Cervical Disc Pathology
}

Jun Ho Lee

Department of Neurosurgery, Kyung Hee University School of Medicine, Seoul, Korea

Corresponding Author: Jun Ho Lee, MD, PhD Department of Neurosurgery, Kyung Hee University Medical Center, 23 Kyung Hee Daero, Dongdaemun-Gu, Seoul 02447, Korea

Tel: $+82-2-958-8405$

Fax: +82-2-958-8385

E-mail: mo09924@khu.ac.kr

Received: May 9, 2016

Revised: July 11, 2016

Accepted: July 20, 2016
The previous reports on the outcome after anterior cervical discectomy without fusion varies among the institutes. However, after careful selection of the patients with isolated cervical soft disc herniation or focal osteophyte formation without instability, subluxation, malalignment, or loss of normal cervical lordosis, the number of the patients that required a delayed fusion procedure could be reduced to less than $1.5 \%$. With these promising results from the literature, the authors have pursued direct removal of disc space pathology through transcorporeal route from the subjects that have met those selection criteria, leaving the majority of the anterior and central disc, anterior longitudinal ligament, and both upper and lower vertebral endplates intact. This selective removal procedure might exclude the fusion surgery-related complications while deterring the development of subsequent instability. Moreover, since it includes an 'oblique' drilling procedure, this might also give a more free access to the region such as high cervical or cervico-thoracic junction.

Key Words: Transcorporeal, Midline cervical disc, Selective decompression

\section{INTRODUCTION}

A variety of effective surgical options exist for the treatment of patients with degenerative disease of the cervical spine. Generally, surgeons preferentially use the anterior approach to the cervical spine and interspaces, which frequently ensued by whole discectomy and fusion. This modality is rather safe and provides the benefit of directly exposing the offending pathology along with the verification of the decompression of the compromised neural structures. However, as with the previous series, the potential complications after fusion surgery would be the graft or instrument failure, longer duration of operation or hospital stay, necessity to wear brace for several months, and possibility of yielding adjacent segment disease. Moreover, placing the grafts or instruments properly over the high cervical or cervicothoracic junction area would not be an easy procedure during the fusion surgery.

The previous reports on the outcome after anterior cervical discectomy without fusion varies among the institutes ${ }^{1,2,5,6)}$. However, after careful selection of the patients with isolated cervical soft disc herniation or focal osteophyte formation with out instability, subluxation, malalignment, or loss of normal cervical lordosis, the number of the patients that required a delayed fusion procedure could be reduced to less than $1.5 \%{ }^{4)}$. With these promising results from the literature, the authors have pursued direct removal of disc space pathology through transcorporeal route from the subjects that have met those selection criteria, leaving the majority of the anterior and central disc, anterior longitudinal ligament, and both upper and lower vertebral endplates intact. This selective removal procedure might exclude the fusion surgery -related complications while deterring the development of subsequent instability. Moreover, since it includes an 'oblique' drilling procedure, this might also give a more free access to the region such as high cervical or cervicothoracic junction.

\section{Goal of Surgery}

The main goal of transcorporeal decompression is to directly address the isolated compressive disc space pathology through limited endplate disruption. This can be combined with fusion or anterior foraminotomy procedure on the other cervical level when the disc space pathologies are involved on the multi-level. 


\section{Treatment Options and Critical Points of Making Decision}

The conventional surgical treatment modality for the isolated cervical disc pathology that most surgeons would choose would be anterior cervical discectomy and fusion. However, getting access to or placing a solid plate and screw along with bone graft down to T2 level even after full decompression would be quite demanding and therefore would require big longitudinal incision along the sternum. Moreover, the combined cervical pathology, as in the second case, would conventionally have required multilevel fusion surgery.

The choice of selective removal of herniated pathology through transcorporeal route would be proper for these patients, as the procedure might adequately decompress the spinal cord while relegating the necessity to perform a complicated surgical approach or multi-level fusion. However, as the authors have described earlier, the subjects with the evidence of cervical instability, subluxation, malalignment, or loss of normal cervical lordosis especially at the corresponding level should be refrained from undergoing this procedure ${ }^{4)}$.

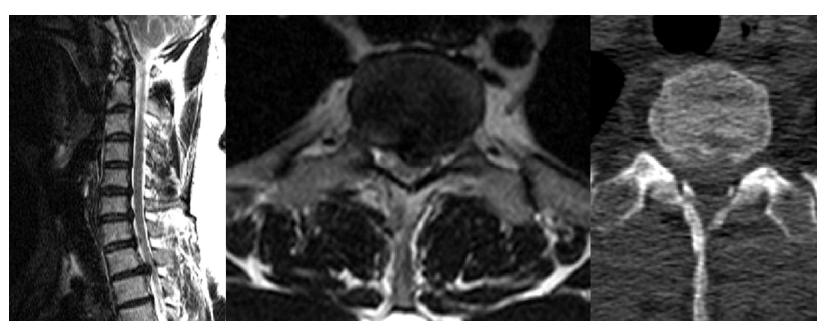

Fig. 1. The preoperative T2-MRI sagittal, axial and CT scan (case \#1) revealed a severely protruded disc herniation combined with small osteophyte on the T1-2 disc level, compromising the left portion of the spinal cord by occupying more than half of the canal diameter at its maximal dimension. The evidence of myelopathy is also definite on the sagittal MRI image.

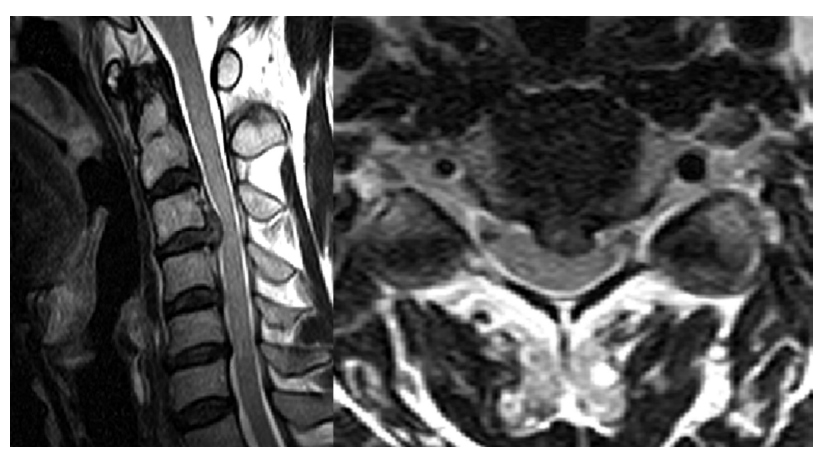

Fig. 2. The preoperative T2-MRI sagittal, and axial scan (case \#2) revealed a protruded disc herniation which have migrated upwardly on the C3-4 disc level, compressing the spinal cord mostly from central.

\section{Positioning and procedures}

The patient is in supine position and is approached from the more compressive and more symptomatic side. Usually a transverse skin incision is made corresponding to the mid-body portion either above or below of the pathologic disc level. Access to the cervical spine is gained by following the standard SmithRobinson approach. After the exposure of the vertebral bodies and ipsilateral longus colli muscle, the longus colli muscle is carefully and minimally displaced laterally just to expose the tip of medial portion of the transverse process. Caspar screws were in zserted at the exact mid-portion of the bodies and using this as a medial retractor, a retractor was placed between the screw and displaced longus colli muscle.

The drilling is started from 3-4 mm above or below the bony endplate on the vertebral body on the either side of the disc space. The drilling depth went further vertically into the bodies until the juntion between the endplate and posterior cortical bone is exposed. Since the procedure is composed of a bit 'oblique' type drilling, care is taken to avoid the violation to the slim wall of bony endplate during the initial phase of the vertical

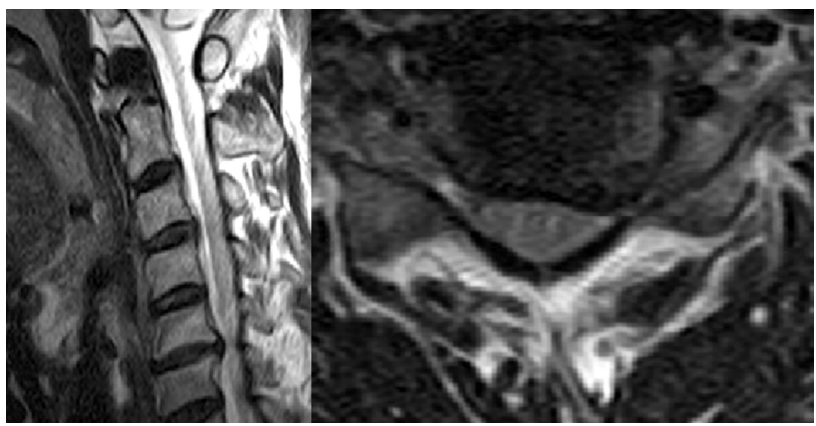

Fig. 3. At the lower C5-6 level, another soft disc herniation compressing the left $\mathrm{C} 6$ nerve root at the foramen is also demonstrated.

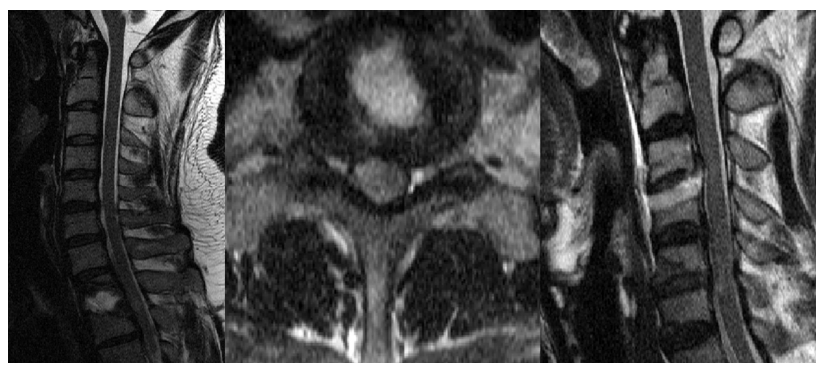

Fig. 4. The postoperative MRI sagittal and axial scan demonstrates the well-decompressed spinal cord at the corresponding level for each case (T1-2 for case \#1 and C3-4 for case \#2) with minimal disruption only on the posterior part of the disc. Note the slight oblique, vertical drilling pathway through the transcorporeal route. 


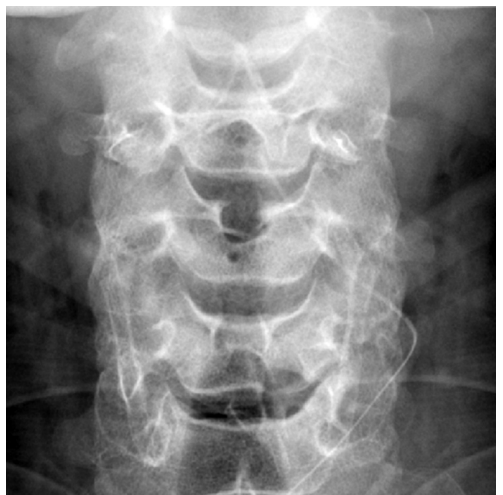

Fig. 5. Also note the surgical defect created by drill hole after the left C5-6 foraminotomy for case \#2.

drilling before the junction has been exposed. Once the posterior limit of the drill hole is reached, the side-cutting burr tip might be used to expand the hole from within. With the careful manipulation and lifting of the remnant posterior cortical bone and posterior longitudinal ligament, the dura would be exposed along with the compressive disc pathology. After the visualization of the compressive pathology, it should be gently removed or drilled off using micro-forcep, blunt hook, laser, or side-cutting drill. Care is taken to not to disrupt the central part of the disc space while using the small sized punch or microcurette. After full decompression, the restoration of the cord pulsation and re-expansion of the dural sac should be confirmed. The adequacy of decompression should be assessed with gentle palpation over the dural sac with blunt hook especially along the direction that coincides with the direction of herniated pathology. Every side of exposed dura should come in contact with bony margin of drill hole once the decompression is achieved sufficiently (Fig. 1-5).

Postoperatively, the patients were advised to wear neck brace 2 to 4 weeks and to avoid excessive movement.

\section{Expected Complications and Results}

The expected surgery-related complication after transcorporeal decompression procedures would be: (1) the cord injury especially during the final stage of vertical drilling, (2) the cerebrospinal fluid leakage during the removal of the compressive pathology, (3) a kyphotic progression of the cervical curvature, and (4) inadequate decompression or recurrence. The cases described above showed no evidence of the complication with complete resolution of their preoperative disabling symptoms.

\section{DISCUSSION}

The purpose of the transcorporeal selective decompression without fusion for the isolated cervical disc pathology would be that achieving adequate decompression through anterior approach while minimizing the effect on the motion segment of the cervical vertebrae ${ }^{7)}$. The conventional anterior cervical discectomy followed by fusion is a safe, direct, and associated with higher success rate for the treatment of cervical discogenic diseases, but it is frequently ensued with potential complications such as loss of a motion segment, graft or instrument failure, or the development of adjacent segment disease. While the most surgeons would apprehend that the performance of cervical discectomy without fusion might yield the consequence of the development of postoperative instability or subsidence of disc space, mere disruption of less than 10 to $15 \%$ of vertebral area through transcorporeal route or partial removal of the posteriorly herniated disc during this selective, decompressive procedure might obviate these concerns ${ }^{3,7)}$. Moreover, there are many proven previous literature supporting the evidence that minor loss in disc height and minor alterations in cervical sagittal curvature after anterior cervical discectomy without fusion do not affect the long-term outcomes ${ }^{1-3,7)}$. Moreover, since it is an 'oblique' procedure, surgeons could use simple incision over the safe cervical region between superior and inferior thyroid artery, thereby avoiding the complicated incision or approaching steps to the high cervical or cervicothoracic junction area(Flg. 1-5), which would not be a readily accessible region during the fusion surgery.

Since it is a very selective procedure working on the very narrow surgical field, chances exist that surgeon might miss the remnant pathology. However, with the recent assistance of the Iso-3-dimensional intraoperative fluoroscopy, these possibilities of the residual pathology have been significantly decreased.

\section{Practical Points}

1. Try to locate the exact center of the anterior vertebral body on the affected disc level during the approach. It should be defined as the midpoint between the both sides of the longus colli muscle or with the aid of the intraoperative fluoroscopy. It is very important to imagine at least 'where' the actual herniated mass is located.

2. Try to create as small defect as possible on the surface of the anterior vertebral body and then widen the drill hole as you reach for the pathology using side-cutting drill. This relative small superficial deficiency would have a higher chance of refilling with reparative new bone formation later.

3. Try not to disrupt the endplate and expose the disc space on the initial phase of the vertical drilling procedure. It is after you define the junction between the endplate and posterior cortical bone that you might disrupt the posterior endplate only to visualize the herniated pathology. Once you reached for the disc space during the early phase of transcorporeal drilling procedure, chances are increasing the you would muchly disrupt the majority of the central nucleus, thereby leading to the development of postoperative instability or disc space subsidence.

4. Gently palpate over the dural surface while you search for the remnant pathology using very soft, micro-blunt hook during the terminal phase of decompression. Once fully decompressed, the dural sac would rapidly expand along with the spinal cord and minor aberrant manipulation could produce direct injury on the anterior aspect of the cord. 


\section{REFERENCES}

1. Benini A, Krayenbuhl H, Bruderl R: Anterior cervical discectomy without fusion. Microsurgical technique. Acta Neurochir (Wien) 61:105-110, 1982

2. Bertalanffy H, Eggert HR: Clinical long-term results of anterior discectomy without fusion for treatment of cervical radiculopathy and myelopathy. Acta Neurochir (Wien) 90:127-135, 1988

3. Choi G, Lee SH, Bhanot A, Chae YS, Jung B, Lee S: Modified transcorporeal anterior cervical microforaminotomy for cervical radiculopathy: a technical note and early results. Eur Spine J 16:
1387-1393, 2007

4. Hadley MN, Sonntag VK: Cervical disc herniations. The anterior approach to symptomatic interspace pathology. Neurosurg Clin N Am 4:45-52, 1993

5. Martins AN: Anterior cervical discectomy with and without interbody bone graft. J Neurosurg 44:290-295, 1976

6. Robertson JT: Anterior removal of cervical disc without fusion. Clin Neurosurg 20:259-261, 1973

7. Snyder GM, Bernhardt AM: Anterior cervical fractional interspace decompression for treatment of cervical radiculopathy. A review of the first 66 cases. Clin Orthop 246:92-99, 1989 